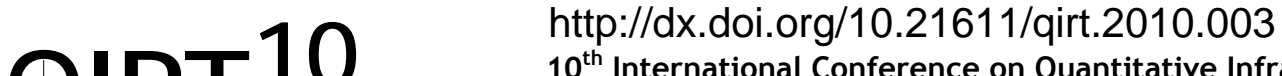 \\ $10^{\text {th }}$ International Conference on Quantitative InfraRed Thermography \\ July 27-30, 2010, Québec (Canada)
}

\section{Multispectral Infrared Face Recognition: a comparative study}

by M. Akhloufi* and A. Bendada*

* Computer Vision and Systems Laboratory, Laval University, Quebec City (Quebec) G1V OA6, Canada, akhloufi@gel.ulaval.ca, bendada@gel.ulaval.ca

\begin{abstract}
Face recognition in the infrared spectrum has attracted a lot of interest in recent years. Many of the techniques used in infrared are based on their visible counterpart, especially linear dimensionality reduction techniques like PCA and LDA. In the thermal infrared spectrum, variations can occur between face images of the same individual due to pose, metabolic, time changes, etc. In this work we introduce the use of non linear dimensionality reduction techniques and a probabilistic Bayesian technique for infrared face recognition. These techniques permit to reduce intrapersonal variation, thus making them very interesting for infrared face recognition.

A comparative study is conducted in order to evaluate the performance of the proposed techniques for infrared face recognition. Experimental results show that the non linear and probabilistic techniques are promising and lead to interesting results in the infrared spectrum.
\end{abstract}

\section{Introduction}

Face recognition is an area of computer vision that has attracted a lot of interest from the research community. A growing demand for robust face recognition software in security applications has driven the development of interesting approaches in this field.

A large quantity of research in face recognition deals with visible face images $[1,2]$. In the visible spectrum the illumination changes represent a significant challenge for the recognition system. Illumination change introduces a lot of errors during the recognition phase. Another challenge for face recognition in the visible spectrum involves the changes in facial expressions. Facial expression can lead to a poor performance of the face recognition system in visible images. To avoid these problems, researchers propose the use of 3D face recognition [3] and infrared face recognition [4,5]. Infrared face recognition is a growing area of research. Many of the techniques used in infrared face recognition are inspired from their visible counterparts. Known techniques used in visible face image recognition are also used with infrared images, like Eigenfaces or Fisherfaces $[4,5]$. More recently in $[6,7,8]$ physiological information extracted from high temperature regions in thermal face images were used in infrared face recognition.

The vast majority of infrared face recognition techniques are based on linear approaches used in visible face recognition. Recent work has been conducted using non linear dimensionality reduction techniques for infrared face recognition with promising results [9]. In this work we introduce a probabilistic Bayesian framework and new non linear subspace learning for face recognition in the infrared spectrum. Different Bayesian techniques were proposed for visible face recognition $[10,11]$. Bayesian techniques and non linear subspace learning can reduce intrapersonal variation which makes them suitable for face recognition. In the infrared spectrum, variations can occur between face images of the same individual due to pose, metabolic, time changes, etc. The proposed approach adapt to these changes in the learning step thus reducing possible errors during the recognition process.

Tests were conducted using two infrared face databases: Equinox multimodal face database [12] and a new infrared multispectral face database we have developed recently in order to evaluate infrared face recognition techniques in a close to real world situations.

\section{Multispectral face databases}

In this work we used the popular Equinox database and a new multispectral database we developed for infrared face recognition tests. 


\subsection{Equinox Multimodal Database}

The Equinox multimodal database [12] is a large collection of face images. These images were acquired using a special setup formed by visible and infrared cameras and a controlled lighting system (frontal, left and right lights). The following modalities are available:

1. Visible (0.4-0.7 microns);

2. Short-wave (SWIR, 0.9-1.7 microns);

3. Long-wave infrared (LWIR, 8-12 microns);

4. Mid-wave infrared (MWIR, 3-5 microns).

The image acquisition was conducted under controlled conditions. Multiple facial expressions, illumination changes and facial images with and without eyeglasses are available.

\subsection{Laval University Multispectral Face Database}

A new multispectral face database was developed in order to evaluate the performance of infrared face recognition techniques. This database has the following spectrums (Fig. 1):

1. Visible (0.4-0.7 microns);

2. Near infrared (NIR, 0.8-0.9 microns);

3. Long-wave infrared (LWIR, 8-12 microns);

4. Mid-wave infrared (MWIR, 3-5 microns).

The images have a higher resolution than equinox (640x480 for visible and NIR images and 640x512 for MWIR images). Image acquisition was conducted in a less controlled environment compared to Equinox. The database contains multiple facial expressions, changes over time, metabolic variations (after temperature change due to exercise) and presence of eyeglasses.

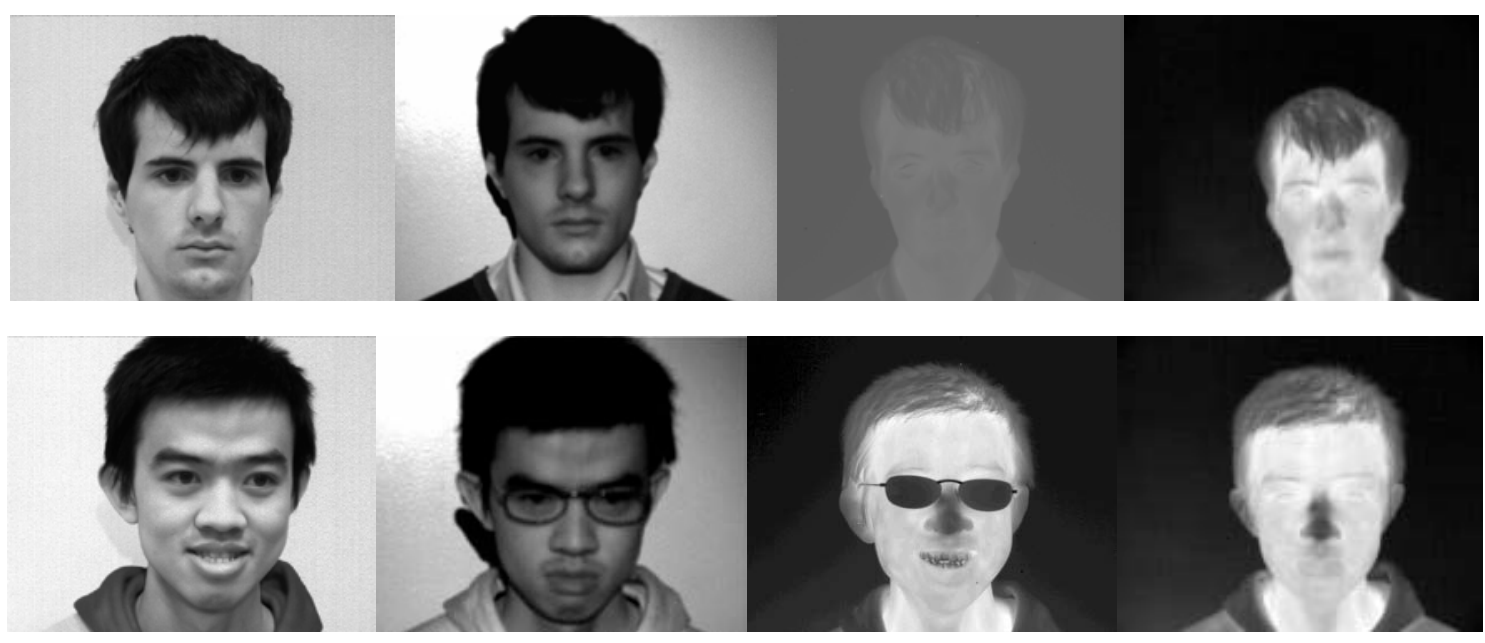

Fig. 1. Face images from Laval University Multispectral Face Database (Visible, NIR, MWIR and LWIR). 


\section{Face extraction and alignment}

For face learning and recognition, the face region was extracted and normalized. The normalization is based on the extracted eyes and mouth positions. The normalized face images can be used for face recognition and for multispectral image fusion. Two different approaches were developed. The first approach operate in visible and active infrared spectrums (NIR and SWIR) and the second approach operates in the thermal infrared spectrums [24].

\subsection{Face extraction in active IR and visible spectrums}

For face extraction in the visible and active IR (NIR and SWIR) spectrums we use the algorithm given in figure 2.

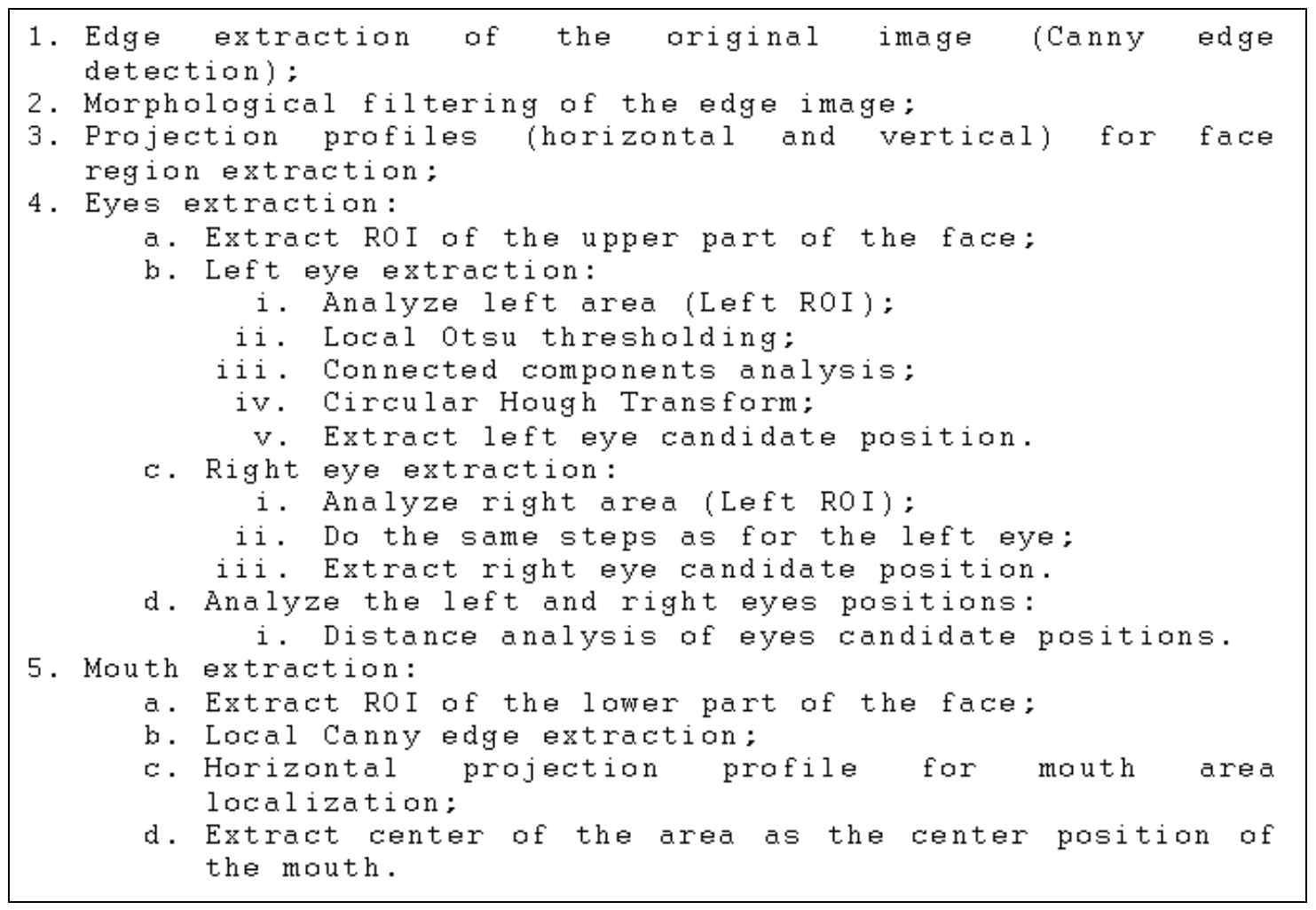

Fig. 2. Pseudo-code for active IR / Visible face extraction algorithm.

\subsection{Face extraction in thermal IR}

For MWIR and LWIR face images, learned thermal data is used in order to segment the face region using an SVM (Support Vector Machines) classifier. In thermal face images the internal eye region show high thermal values. This property is used in order to extract the eyes positions. For face extraction in the thermal IR (MWIR and LWIR) spectrums we use the algorithm given in figure 3 . 


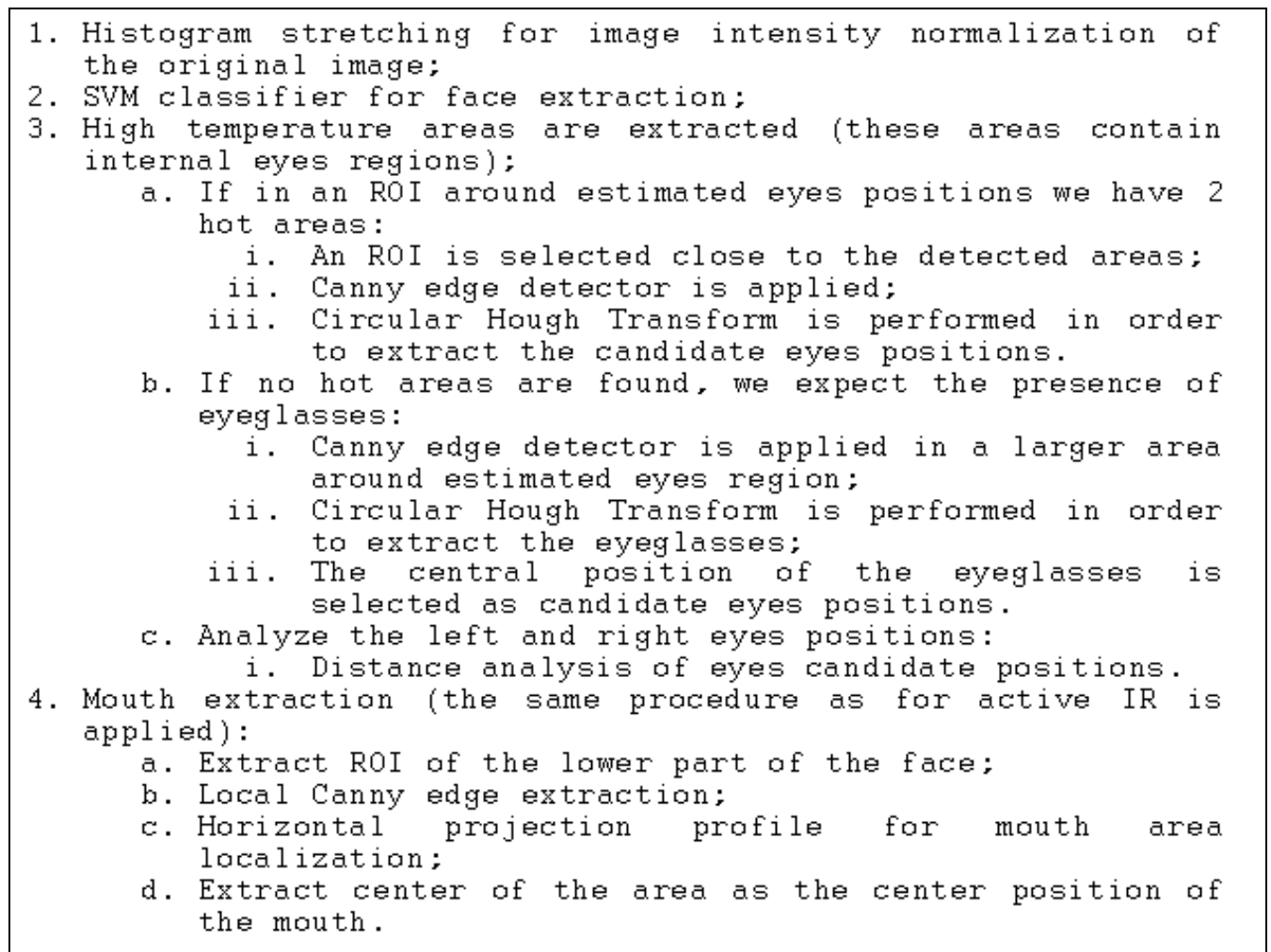

Fig. 3. Pseudo-code for thermal IR face extraction algorithm.

\subsection{Face alignment}

The obtained positions of eyes and mouth give three positions that are used to extract the parameters of an affine transformation matrix to a normalized triangle position in a $128 \times 128$ image (Fig. 4). A region of interest is then extracted relative to the normalized triangle. This region keeps the most interesting face areas for further processing. The extracted face images are used for face learning, face recognition and multispectral data fusion (after this step all the obtained images are co-aligned).

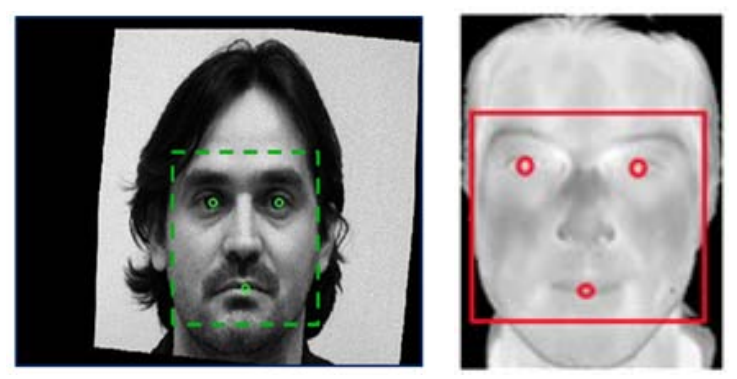

Fig. 4. Example of face alignment and extraction in visible and MWIR 


\section{Face recognition}

\subsection{Bayesian face recognition}

The proposed Bayesian approach is based on the work described in [11]. A combination of PCA technique and a Bayesian Maximum Likelihood (ML) is used in order to classify the face image. The Bayesian approach uses a probabilistic measure of similarity based on a Bayesian Maximum Likelihood (ML) analysis of image differences [10], [11]. The PCA is used for the projection of differenced images in two subspaces: intrapersonal and extrapersonal. An intrapersonal subspace are learned from face images of the same person using the PCA face recognition approach, while extrapersonal subspaces is learned from faces of multiple individuals as in the standard eigenfaces technique (PCA).

The projection in the two sets of learned eigenfaces is used to compute and compare the likelihoods $P\left(\Delta \mid \Omega_{l}\right)$ and $P\left(\Delta \mid \Omega_{E}\right)$. The image is considered to belong to the same person if $P\left(\Delta \mid \Omega_{l}\right)>P\left(\Delta \mid \Omega_{E}\right)\left(\Omega_{l}\right.$ and $\Omega_{E}$ represent respectively the intrapersonal and extrapersonal eigenfaces. $\Delta$ is the difference image).

\subsection{Non linear subspace learning}

Face learning and recognition techniques based on linear and non linear dimensionality reduction were implemented. Dimensionality reduction techniques are a set of mathematical techniques used for representing available data in a low dimensional space. The obtained representation is a compressed version of the original data with the most important characteristics preserved for further processing. Dimensionality reduction seek to represent a set of data as a p-dimensional manifold embedded in an $m$-dimensional space (with $p<m$ ) [13]. Non linear dimensionality reduction for subspace learning was implemented in order to obtain a face space that best detects the essential manifold structure for face recognition. In the infrared spectrum, variations can occur between face images of the same individual due to pose, metabolic, time changes, etc. In the visible and active infrared spectrums variations can occur due to facial expressions, pose, etc. By using non linear techniques, unwanted variations resulting from these changes may be eliminated or reduced. The following approaches are available $[9,24]$ :

- $\quad$ Linear techniques $[19,20]$ : the most popular techniques are implemented. Eigenfaces (PCA) technique followed by Fisherfaces (LDA) technique. These techniques serve as a reference for performance comparison with other techniques.

- $\quad$ Global kernel based non linear techniques [14,15]: Kernel based subspace learning techniques have been used in order to overcome the limitations of linear mapping. Kernel-PCA and Kernel-LDA are available in the framework.

- Local non linear techniques [15-18,20-23] : in this work two local non linear techniques are implemented for multispectral face recognition, Linear Graph Embedding (LGE) and Locality Preserving Projection (LPP), and their tensor extension Tensor-LGE (TLGE), and Tensor-LPP (TLPP) [23]. LGE and LPP are a non-linear dimensionality reduction technique. Their assumption is that each data point and its neighbors lie on or close to a locally linear patch of the manifold. These techniques find an embedding that preserves local information. The aim of these techniques is to represent the data in a lower dimensional subspace while preserving the local structure of the original image space. Also, a recently introduced local technique is implemented [21]: Locality Sensitive Discriminant Analysis (LSDA). LSDA is based on LDA and permits to avoid LDA disadvantages when little samples are available. It aims to discover a local manifold structure and finds a projection which maximizes the margin between data points from different classes at each local area.

- $\quad$ Local kernel based non linear techniques [15,17,22]: Kernel-LGE (KGE) and Kernel-LPP (KLPP).

\section{Experimental results}

Experimental tests were conducted using the proposed framework in order to evaluate the performance of multispectral face recognition on two databases: Equinox and Laval University. The images were extracted and aligned using the proposed techniques.

The databases face images were split in two independent sets. One set used for subspace learning and the second set for subspace face recognition. Learning was conducted using the proposed algorithms : PCA, KPCA, LGE, TLGE, KGE, LDA, KDA, LPP, TLPP, KLPP, LSDA and Bayesian technique. Learning was conducted on the extracted face images in different spectrums. For face recognition, 10 tests per scenario was conducted using randomly chosen 100 face images for each test (total of 1000 recognition tests per scenario). The tested face images contain variations like: facial expressions, pose, eyeglasses, facial hair, etc. The obtained results are given in tables 1 and 2 below. 
For equinox database (Table 1), LSDA was the best performing, followed by LDA and LPP. Higher results were obtained for short wave infrared (SWIR) face images attaining 76\% success rate with LDA, followed closely by the visible spectrum with $75 \%$ using LSDA.

For Laval University (LUMF) database (Table 2), LDA give the best performance followed by Bayesian technique, and LSDA. Higher results were obtained for visible spectrum face images attaining 94\% success rate with LSDA followed closely by the near infrared (NIR) and mid wave infrared (MWIR) with 65\% using LGE and LDA respectively. The LDA and Bayesian technique performance can be explained by the availability of more face images for each individual in the intrapersonal learning process in Laval University database.

The cumulative best scores results of the first 5 matches show an important increase in the recognition performance for the two databases. The obtained results show that the correct match is always within the first $\boldsymbol{N}$ results. This observation is used in a new proposed scheme we are developing for a multi-stage high performance automatic face recognition system.

Table 1. Results for Equinox Database

\begin{tabular}{|l||c|c|c|c||c|c|c|c|}
\cline { 2 - 9 } \multicolumn{1}{c|}{} & \multicolumn{4}{c||}{ First match } & \multicolumn{3}{c|}{ Cumulative best scores } \\
\cline { 2 - 9 } \multicolumn{1}{c||}{} & Visible & SWIR & MWIR & LWIR & Visible & SWIR & MWIR & LWIR \\
\hline PCA & $16 \%$ & $29 \%$ & $32 \%$ & $25 \%$ & $30 \%$ & $77 \%$ & $60 \%$ & $43 \%$ \\
\hline KPCA & $0 \%$ & $6 \%$ & $3 \%$ & $7 \%$ & $14 \%$ & $30 \%$ & $16 \%$ & $15 \%$ \\
\hline LGE & $42 \%$ & $68 \%$ & $6 \%$ & $2 \%$ & $80 \%$ & $78 \%$ & $38 \%$ & $6 \%$ \\
\hline TLGE & $25 \%$ & $60 \%$ & $2 \%$ & $3 \%$ & $53 \%$ & $85 \%$ & $28 \%$ & $20 \%$ \\
\hline KGE & $0 \%$ & $5 \%$ & $0 \%$ & $2 \%$ & $12 \%$ & $19 \%$ & $22 \%$ & $19 \%$ \\
\hline LDA & $2 \%$ & $76 \%$ & $0 \%$ & $5 \%$ & $14 \%$ & $91 \%$ & $9 \%$ & $8 \%$ \\
\hline KDA & $0 \%$ & $5 \%$ & $0 \%$ & $0 \%$ & $9 \%$ & $19 \%$ & $26 \%$ & $12 \%$ \\
\hline LPP & $67 \%$ & $66 \%$ & $18 \%$ & $18 \%$ & $88 \%$ & $89 \%$ & $32 \%$ & $32 \%$ \\
\hline TLPP & $23 \%$ & $25 \%$ & $10 \%$ & $2 \%$ & $45 \%$ & $72 \%$ & $33 \%$ & $17 \%$ \\
\hline KLPP & $4 \%$ & $4 \%$ & $3 \%$ & $3 \%$ & $17 \%$ & $14 \%$ & $16 \%$ & $14 \%$ \\
\hline LSDA & $75 \%$ & $68 \%$ & $40 \%$ & $46 \%$ & $85 \%$ & $72 \%$ & $64 \%$ & $69 \%$ \\
\hline Bayes & $32 \%$ & $70 \%$ & $39 \%$ & $41 \%$ & $50 \%$ & $71 \%$ & $54 \%$ & $62 \%$ \\
\hline
\end{tabular}

Table 2. Results for Laval Univ. Multispectral Database

\begin{tabular}{|l||c|c|c|c||c|c|c|c|}
\cline { 2 - 9 } \multicolumn{1}{c|}{} & \multicolumn{4}{c||}{ First match } & \multicolumn{3}{c|}{ Cumulative best scores } \\
\cline { 2 - 9 } \multicolumn{1}{c|}{} & Visible & NIR & MWIR & LWIR & Visible & NIR & MWIR & LWIR \\
\hline PCA & $39 \%$ & $10 \%$ & $35 \%$ & $42 \%$ & $71 \%$ & $61 \%$ & $61 \%$ & $70 \%$ \\
\hline KPCA & $3 \%$ & $5 \%$ & $5 \%$ & $5 \%$ & $16 \%$ & $21 \%$ & $21 \%$ & $20 \%$ \\
\hline LGE & $80 \%$ & $65 \%$ & $26 \%$ & $19 \%$ & $93 \%$ & $51 \%$ & $51 \%$ & $52 \%$ \\
\hline TLGE & $67 \%$ & $14 \%$ & $32 \%$ & $11 \%$ & $87 \%$ & $51 \%$ & $51 \%$ & $42 \%$ \\
\hline KGE & $8 \%$ & $1 \%$ & $0 \%$ & $2 \%$ & $13 \%$ & $9 \%$ & $9 \%$ & $9 \%$ \\
\hline LDA & $86 \%$ & $60 \%$ & $65 \%$ & $54 \%$ & $96 \%$ & $78 \%$ & $78 \%$ & $76 \%$ \\
\hline KDA & $2 \%$ & $4 \%$ & $2 \%$ & $2 \%$ & $13 \%$ & $11 \%$ & $11 \%$ & $12 \%$ \\
\hline LPP & $66 \%$ & $31 \%$ & $52 \%$ & $44 \%$ & $86 \%$ & $65 \%$ & $65 \%$ & $58 \%$ \\
\hline TLPP & $35 \%$ & $29 \%$ & $24 \%$ & $16 \%$ & $76 \%$ & $44 \%$ & $44 \%$ & $38 \%$ \\
\hline KLPP & $2 \%$ & $3 \%$ & $8 \%$ & $1 \%$ & $12 \%$ & $17 \%$ & $17 \%$ & $12 \%$ \\
\hline LSDA & $94 \%$ & $37 \%$ & $55 \%$ & $45 \%$ & $98 \%$ & $66 \%$ & $66 \%$ & $53 \%$ \\
\hline Bayes & $80 \%$ & $62 \%$ & $65 \%$ & $50 \%$ & $88 \%$ & $70 \%$ & $86 \%$ & $70 \%$ \\
\hline
\end{tabular}




\section{Conclusion}

In this work we presented an evaluation of different face recognition technique for multispectral face recognition. A new face extraction an normalization techniques are introduced and used to normalize the face images in different spectrums. Different face learning and recognition techniques are implemented, they are based on linear and non linear subspace learning and recognition techniques. Also available, are LBP-like descriptors are available and can be used for image preprocessing prior to face learning and recognition. Finally, multi-scale fusion algorithms are also implemented in this framework and can be used for the evaluation of fusion schemes in multispectral face recognition.

Tests were conducted in order to evaluate the performance of the implemented face recognition techniques in different spectrums. LDA, LSDA and Bayesian techniques were the best performing. Cumulative score tests were performed. They show that when we search the face image within the first $N$ matches, we obtain higher scores. This strategy is part of ongoing research for implementing a multi-stage automatic face recognition system permitting an increase in face recognition performance.

Future work includes the use of texture space analysis and multi-scale fusion.

\section{REFERENCES}

[1] A.K. Jain, Handbook of Multibiometrics, Springer, 2006.

[2] A.K. Jain, P. Flynn, and A.A. Ross, Handbook of Biometrics, Springer, 2007.

[3] A. Bronstein, M. Bronstein, and R. Kimmel., "Three-dimensional face recognition", International Journal of Computer Vision, vol. 64, pp. 5-30, 2005.

[4] S. Kong, J. Heo., B. Abidi, J. Paik, and M. Abidi, "Recent advances in visual and infrared face recognition: a review", Computer Vision \& Image Understanding, vol. 97, pp. 103-135, 2005.

[5] M.A. Akhloufi, A. Bendada, and J.C. Batsale, "State of the art in Infrared face recognition", QIRT Journal, vol.5, n.1, pp.3-26, June 2008.

[6] P. Buddharaju, I.T. Pavlidis and P. Tsiamyrtzis, "Pose-Invariant Physiological Face Recognition in the Thermal Infrared Spectrum", IEEE Conference on Computer Vision and Pattern Recognition Workshop, New York , pp. 5353, 2006.

[7] M.A. Akhloufi, and A. Bendada, "Thermal Faceprint: A new thermal face signature extraction for infrared face recognition", Proceedings of the 5th Canadian Conference on Computer and Robot Vision (CRV 2008), Windsor, ON, Canada, May 2008.

[8] M.A. Akhloufi, A. Bendada, "Infrared Face Recognition Using Distance Transforms", Proceedings of the 5th International Conference on Image and Vision Computing (ICIVC 2008), Vol.30, pp. 160-163, Paris, France, July 0406, 2008.

[9] M. A. Akhloufi, A. Bendada, and J.C. Batsale, "Multispectral face recognition using non linear dimensionality reduction", Proceedings of SPIE Visual Information Processing XVIII conference, Volume 7341, SPIE Defense, Security and Sensing, 7341-19, Orlando, Florida, USA, 2009.

[10] B. Moghaddam, C. Nastar, and A. Pentland, "Bayesian face recognition using deformable intensity surfaces", IEEE Computer Society Conference on Computer Vision and Pattern Recognition (CVPR'96), San Francisco, CA, USA, pp. 638-645, 1996.

[11] B. Moghaddam, T. Jebara, and A. Pentland, "Bayesian Face Recognition", Pattern Recognition, Vol. 33, Issue 11, pp. 1771-1782, 2000.

[12] Equinox, "Multimodal face database", http://www.equinoxsensors.com/products/HID.html, last visited August 2009.

[13] L., Journaux, I. Foucherot, and P., Gouton, "Operational Comparison of Dimensionality Reduction Techniques applied on Multispectral Satellite Images," Proc. 4th Int. Conf. Signal-Image Technology \& Internet-Based Syst., 476-484, 2006.

[14] M. Yang, N., Ahuja, and D. Kriegman, "Face Recognition Using Kernel Eigenfaces", Proc. of IEEE ICIP, 37-40 (2000).

[15] Q. Liu, R. Huang, H. Lu, and S. Ma, "Kernel-based Optimized Feature Vectors Selection and Discriminant Analysis for Face Recognition". In Proc of ICPR'02, 362-365, 2002.

[16] Y. Pang, L. Zhang, Z. Liu, N. Yu, and H. Li, "Neighborhood Preserving Projections (NPP): A Novel Linear Dimension Reduction Method". In Proc. of International Conference on Intelligent Computing (ICIC 2005), pp. 23-26, 2005.

[17] X. He, S., Yan, Y., Hu, and H., Zhang, "Learning a Locality Preserving Subspace for Visual Recognition", Proc. of the IEEE Int. Conf. on Comp. Vision (ICCV 2003), 385-393 (2003).

[18] P. Niyogi, "Laplacian Eigenmaps for Dimensionality Reduction and Data Representation", Neural Computation, 15, 1373-1396 (2003).

[19] M. Turk, and A. Pentland, "Face recognition using eigenfaces", In Proc. of the IEEE Comp. Soc. Conf. on Comp. Vision and Patt. Recog. (CVPR'91), 586-591, 1991.

[20] A. Martinez, and A.C. Kak, "PCA versus LDA". IEEE Trans. on PAMI, 23 (2), 228-233, 2001. 
[21] D. Cai, X. He, K. Zhou, J. Han, and H. Bao, H. "Locality sensitive discriminant analysis", In Proceedings of the 20th international Joint Conference on Artifical intelligence, pp. 708-713, Hyderabad, India, January 2007.

[22] D. Cai, X. He, Y. Hu, J. Han and T. Huang, "Learning a Spatially Smooth Subspace for Face Recognition", Proc. IEEE Conf. Computer Vision and Pattern Recognition Machine Learning (CVPR'07), pp. 1-7, July 2007.

[23] X. He, D. Cai and P. Niyogi, "Tensor Subspace Analysis". Advances in Neural Information Processing Systems 18 (NIPS 2005), pp. 499-506, Vancouver, Canada, 2005.

[24] M.A. Akhloufi, and A. Bendada, "A new framework for face recognition in and beyond the visible spectrum", Proc. of the 2010 IEEE International Conference on Systems, Man, and Cybernetics (SMC 2010), October 2010. 